\section{Venous air embolism during scalp dissection in a case of cerebellar haemangioblastoma}

\section{Surya Kumar Dube, Vivek Bharti Sharma, Shailendra Kumar, Girija Prasad Rath}

Sir,

Venous air embolism (VAE) is the entrainment of air from the operative field or other communication with the environment into the venous vasculature producing systemic effects. ${ }^{[1]}$ We report a case of VAE during scalp dissection in a patient undergoing craniotomy in sitting position for cerebellar haemangioblastoma. A 17-year-old, $40 \mathrm{~kg}$, American Society of Anaesthesiologists physical status I, male patient presented with gait instability for 1 year and headache in occipital region for 9 months. The patient was diagnosed to have cerebellar haemangioblastoma [Figure 1] and was posted for excision in sitting position. There was no history of any respiratory or cardiovascular abnormality. His routine blood investigations, electrocardiogram, and chest X-ray were within normal limits. The patient was premedicated with intramuscular glycopyrollate $0.2 \mathrm{mg} 1$ hour before induction of anaesthesia. A general endotracheal

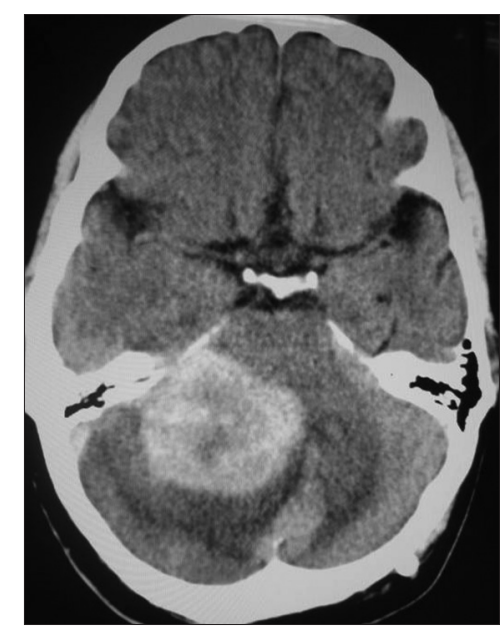

Figure 1: Contrast enhanced computed tomographic scan of the head showing tumour mass in the posterior fossa (cerebellar haemangioblastoma)

anaesthesia with fentanyl, propofol, sevoflurane, vecuronium and nitrous oxide and oxygen (2:1 ratio) was used. Central venous cannulation (right subclavian vein) and intraarterial cannulation was performed following induction of anaesthesia. The patient was preloaded with 1 litre of crystalloid solution to ensure a central venous pressure of $8 \mathrm{mmHg}$, before the patient was made seated. The vital parameters of the patient remained unremarkable after sitting position. But soon after the surgeons started dissection of the scalp and pericranium, a sudden fall in end tidal carbon dioxide $\left(\mathrm{E} \mathrm{tCO}_{2}\right)$ from $34 \mathrm{mmHg}-20 \mathrm{mmHg}$ and blood pressure from

Department of Neuroanaesthesiology, All India Institute of Medical Sciences, New Delhi, India 
120/73 mmHg-80/43 mmHg was observed. The rapidity of the event leads us to assume VAE to be the most probable cause of the event. Immediately, the patient's lung was ventilated with $100 \%$ oxygen. The surgery was briefly interrupted and the operative field was covered with normal saline. In view of hypotension $500 \mathrm{ml}$ of hydroxyl ethyl starch and $10 \mathrm{mg}$ of mephenteramine (in $5 \mathrm{mg}$ increments) were administered; simultaneously, approximately $40 \mathrm{ml}$ of air was aspirated through the central venous catheter. Within a period of 15 minutes, the patient's haemodynamic parameters and $\mathrm{EtCO}_{2}$ returned back to normal. Rest of the surgery was uneventful.

The prerequisites for the entry of gas into the venous system are incision of non-collapsible veins such as diploic veins, emissary veins or dural venous sinuses in presence of sub-atmospheric pressure (e.g., during surgeries performed with patient in sitting position). ${ }^{[1]}$ $\mathrm{EtCO}_{2}$ is a semi-quantitative and non-invasive monitor of intermediate sensitivity for the detection of VAE. ${ }^{[2]}$ However, decrement of $\mathrm{EtCO}_{2}$ is not specific to VAE. Acute hypotension and pulmonary embolism due to any cause can also lead to a decrease in $\mathrm{EtCO}_{2}{ }^{\left[{ }^{[3]}\right.}$ We ruled out pulmonary embolism as to be the cause of the event as there were no predisposing factors associated in our patient. The other possibility was acute hypotension due to sudden blood loss, which may happen after rupture of venous sinuses during the initial part of the surgery. Acute hypotension can cause fall in $\mathrm{EtCO}_{2}$ but unlike
VAE the fall is usually gradual. ${ }^{[3,4]}$ So we considered the aetiology of the above event to be VAE and managed as per the standard management protocol. ${ }^{[2]}$ With this case report, we want to emphasise that VAE can occur through occipital diploic veins even during dissection of scalp and pericranium in posterior fossa surgery with patient in sitting position. In the event of sudden and sustained fall in $\mathrm{EtCO}_{2}$ during surgery in sitting position, VAE should be the first diagnosis even at the initial stages of surgery.

\section{REFERENCES}

1. Muth CM, Shank ES. Gas embolism. N Engl J Med 2000;342:476-82.

2. Mirski MA, Lele AV, Fitzsimmons L, Toung TJ. Diagnosis and treatment of vascular air embolism. Anesthesiology 2007;106:164-77.

3. Bithal P, Dash HH, Vishnoi N, Chaturvedi A. Venous air embolism: Does the site of embolism influence the hemodynamic changes? Neurol India 2003;51:370-2.

4. Hatle L, Rokseth R. The arterial to end tidal carbon dioxide tension gradient in acute pulmonary embolism and other cardiopulmonary disease. Chest 1974;66:352-7.

\begin{tabular}{|l|l|}
\hline \multicolumn{2}{|c|}{ Access this article online } \\
\hline Quick Response Code: & Website: \\
\hline & www.jnaccjournal.org \\
\cline { 2 - 3 } & \\
\hline
\end{tabular}

